\title{
Modified Energy Efficient with ACO Routing Protocol for MANET
}

\author{
${ }^{1}$ Uppalapati Srilakshmi, ${ }^{2}$ Garnepudi Parimala, ${ }^{3}$ Addanki Sathyavani \\ ${ }^{1,2}$ Department of CSE, VFSTR Deemed to be University, Vadlamudi, Guntur, A.P, India. \\ ${ }^{3}$ Department of CSE, Chalapathi Institute of Technology, A.R.Nagar, Mothadaka, Guntur, A.P, India. \\ ${ }^{1}$ srilu.uppalapati@gmail.com, ${ }^{2}$ garnepudiparimala@gmail.com, ${ }^{3}$ sathyavani.addanki@gmail.com
}

Article History: Received: 10 November 2020; Revised: 12 January 2021; Accepted: 27 January 2021; Published online: 05 April 2021

Abstract: Due to the specific design of the network upon the requirements of the demands as well as the situation
at which the setting up of a physical network is impossible, a significant role is played by mobile ad-hoc network
(MANET) in the applications of military. Various critical tasks like robust \& dynamic military workstations,
devices as well as smaller sub-networks within the battle field are handled by the presented network type controls
the infrastructure less communication. A highly demand of the efficient routing protocol's design is existing which
ensures safety as well as reliability to transmit the extremely vulnerable as well as the secret military data within
the defense networks. The designing of an energy efficient layer routing protocol within the network on behalf of
the military applications is done in this method and the simulation is done with the help of a novel cross layer
approach of design for increasing the reliability as well as the lifetime of the network. However, the optimum path
selection is not allowed by PDO-AODV technique. Therefore, a novel ACO-DAEE (Ant colony optimization with
delay aware energy efficient) to select an optimum path as well as to mitigate the delay time within the network
system is proposed. Maintaining the optimum paths within the network while transmitting the data effectively is
the major objective. With respect to packet delivery ratio, end to end delay, and throughput, it is indicated by the
simulation outputs that the performance of ACO-ADEE is quite good. The efficiency of this technique is verified
in the simulation outcomes using NS2 software. Keywords: MANET, PDO-AODV, ACO-DAEE, Forwarder Selection Function (FSF), Second Hand Information (SHI)

\section{Introduction}

The development of the huge technical transformation of wireless communication was done within MANETs. Excluding the fixed infrastructure, the construction of MANETs [1] are possessing ideal networking structure. The efficiency of the network remains impressive with respect to the transmission, throughput as well as reliability due to higher dynamic, extreme mobile and self-configurable behaviour of the individual nodes. The constrained battery power that is reduced in a gradual way due to the additional processing like residual battery within the node, and while transmitting as well as receiving the hello messages, control information, data packets, forwarding packets and messages, processing routing logic and so on are used to equip the nodes within the mobile. Several investigators suggested various routing protocols for optimizing the network efficiency that includes power consumption, delay management techniques and so on. In particular, 2 kinds of routing protocols are present in MANET where, one kind is table driven and the other depends upon the demand. A significant role is played by MANETs within the battle fields as well as the disaster circumstances like network deployment, higher safety efforts within the network, transmission of an end to end, connecting the mobile excluding the failure in addition to the anti-jamming scheme [2]. Excluding the failures in the links although within microsecond level, the overall network activities should be performed in a spontaneous way. For obtaining most recent data, or the commands from the chief or for discussing earlier to an activity, the soldiers throughout the real time battle must stay in connection with one another continually. Sustaining the connection is quite complicated due to the fact that the satellite signals cannot penetrate the caves or dense forest or under sea.

The immediate communication service is supported by an efficient as well as an on-demand protocol called as ad hoc on demand distance vector routing (AODV) protocol in MANET [3]. A well-developed enhanced AODV approach is presented by the authors. Broadcasting of a RREQ message is done by the source within AODV function while a transmitted want to send certain information towards the destination. The establishment of the path is done with respect to the logic executed within a specific approach in which the RREQ travels from hop to hop throughout consecutive nodes. Similarly, the creation of a path is done and the routing table stores the additional reverse route which is exactly opposite to the routing direction and this method is useful in creating a forward route on behalf of the data packets. Although certain additional nodes stay inactive for longer periods exclusive to certain packet forwarding or processing tasks, the end-to-end delay is impacted widely and the routes placed within the centre of the network becomes overburdened within this traditional operation mode. 
The implementation of the traditional routing technique where the shortest path is found rather than implementing a balanced path is unsuitable on behalf of MANET which is a magnificent network. Considering a cross layer approach of the power efficiency using the load balanced protocol development on behalf of MANET is motivated by the power deficiency problem as well as the unfair load balancing amid the MANET nodes.

\section{Related Works}

Various intelligent methods that are upon the basis of energy efficiency, delay management and upon the cross layer architecture were proposed by the authors from the past few years. An organized supplement build approach is proposed by Agbaria [4], et al. where, the dynamic scheduling, resource management, velocity, multipath search for providing real-time as well as QOS requirement of a MANET are assumed. An effective method is proposed by Sivakumar and Duraiswamy [5] that supports the Quality of Service (QoS) with the implementation of the loaddistribution as well as the congestion avoidance routing technique. The cost parameters upon the basis of the link loads is computed by the abovementioned method. The congestion is avoided with the transmission of the traffic by using the links which has the less loads. An energy effective routing is proposed by Srivastava and Daniel [6] for improving the link application with the equalization of the energy consumption amid the underutilised as well as exploited features. Certain major reasons such as residual energy, bandwidth, load and hop count for route discovery are dealt by the abovementioned method. The ant colony based load balanced technique within MANET is presented by Ahmed [7], et al. With the establishment of the link using the resource scheduling issue, the analysing of the route is done. Depending upon the mobility of the node, the adjustment of the size is done by this method and the time taken got transmission is reduced.

PC-AODV is presented by MadhanMohan \& Selvakumar [8], which is an additional cross-layer designing method where the power controlling schemes are used for sending the data as well as the control packets of data link layer as well as the network layer. The construction of several routing inflows is done in this method in accordance with the left power level within the nodes. The selection of a path is done with respect to the essential power level while finding a route. The incorporation of the power level logic is done within the route discovery as well as the maintenance stages using this method.

The application of several power levels (PL) is done using various packets in accordance with the values of the routing table. Therefore, power level compatibility is present within the either layers. The energy is consumed in a lower amount as well as possess high packet delivery ratio by this technique. An additional layered method is implemented that improves the effectiveness of power within MANET [9] and it is unlike the traditional designing mode. Moreover, the cross-layer communication is geared among the 3 significant layers such as physical, MAC and network layer. The transmission power is augmented by implementing a novel technique named as cross layer power control (CLCP) with the help of an improved technique where the suitable route amid 2 nodes is found. This method is simulated using NS2 where optimum results are shown. Rath \& Pattanayak [10] performed an indetail investigation upon the real time MANET protocols. Likewise, Pattanayak \& Rath [11] investigated the mobile agent intruder detection system using the delay as well as the difficulties in power.

\section{Proposed framework}

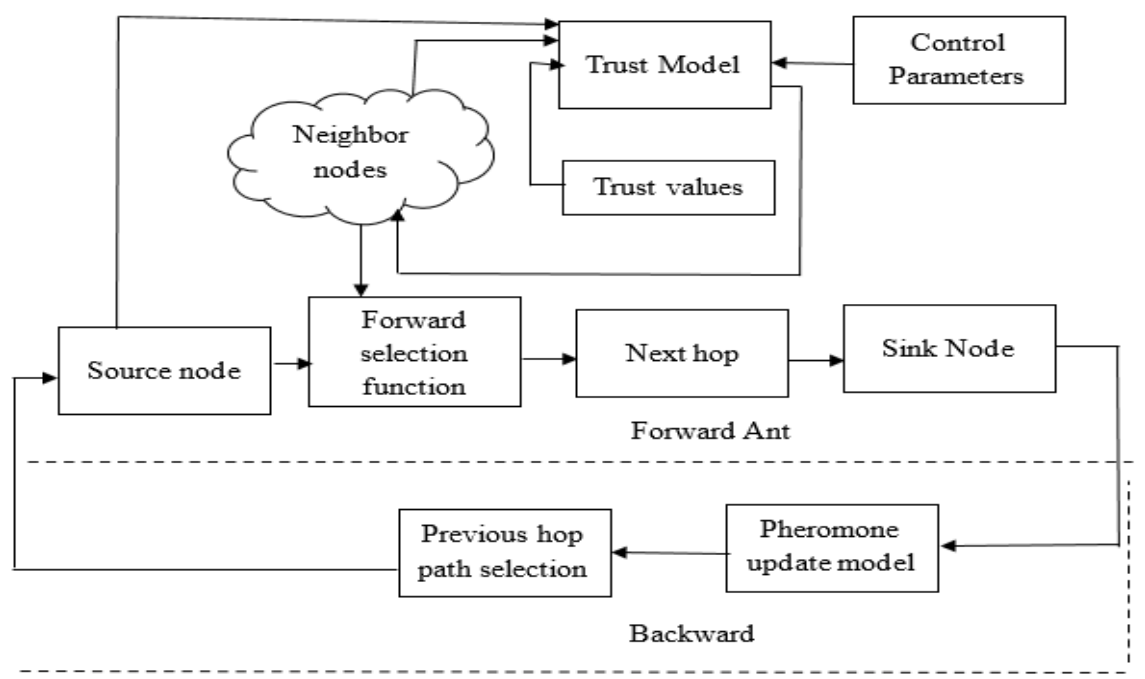

Figure 1. ACO based efficient routing framework 
ACO Based Efficient Routing protocol is presented in this section on behalf of MANET using the Distance, Energy, as well as Link quality. Figure 1 shows this model in which Trust Model, Optimal Forwarder Selection Function (FSF) as well as Improved Pheromone Update Model are included.

A. Trust model

With the help of data of the individual communication with the respective neighbors, the rating of every node is done within this Trust Model. First Hand Information (FHI) is the notation given to it in literature. The individual interaction with the neighbors is collected by the nodes and moreover the node that is rated is assumed as indirect communication. Second Hand Information (SHI) is the notation given to the rated data collected out of the neighbors. The distribution of the simulation time is done within ' $n$ ' slots where two sub-periods namely Forwarding and Monitoring Interval, TFMI followed by Update Interval TUPI are included in every single slot and is shown in Table1.

Table 1. Simulation Period Slots

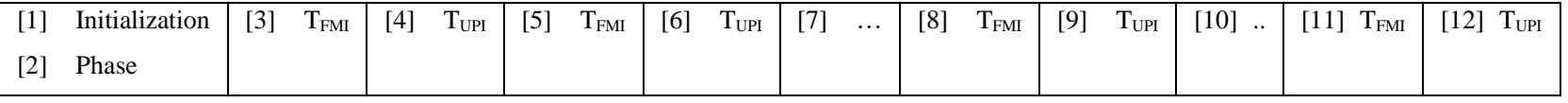

\section{B. Forwarder Selection Function}

For selecting the optimum additional neighbors for forwarding the packets towards the sink node is done by a probability function implemented at each node together with the path from the source towards the sink node within the network named as Forwarder Selection Function. A best path must be selected by the Forwarder Selection Function from the source towards the destination for forwarding the packets for improving the lifetime of the network with the balancing of the energy between the nodes within a network. Moreover it ensures the nodes no to be depleted rapidly as well as the energy of the node is guaranteed by the selection of the high standard links along the path that the energy wastage is avoided because of the frequent retransmissions.

The FSF is implemented by considering the abovementioned reasons which is a probability operation for selecting the optimal forwarder nodes amongst the neighbor nodes of the existing node upon the basis of Pheromone Trail (PT) as well as heuristic function that involves 2 portions that represent the Node Energy level (EN) \& node link quality (LP) functions. The application of the pheromone deposited upon the path amid the nodes that considers Energy, distance and link quality along the path from the source to the destination is represented by Pheromone Trail (PT). Particularly, the optimal quality path from source node towards the destination with respect to energy, distance as well as link quality is represented by high PT. the energy level of the neighboring node is represented by Node Energy (EN) operation and the link quality amid the current as well as the neighboring node is represented by the Link quality (LP) operation.

A probability function where the optimal path from the source towards the destination for forwarding the packets with several goals is referred as Forwarder Selection Function.

- To deliver a safe reliable path from source towards sink in excluding the insider attacks,

- To enhance the Lifetime of the network with balancing the energy amongst the nodes within the network for ensuring that some nodes alongside the path doesn't depletes rapidly (causing Network disconnections or partitioning)

- Simultaneously, choosing good quality links alongside the path for guaranteeing that energy wastage of node is not done because of continual retransmissions.

- $\quad$ Furthermore, shorter path's selection involves least number of nodes causing additional energy saving because of less nodes that takes place in forwarding the packet.

Forwarder Selection Function, the definition of FSF $\left(n_{i}, n_{j}\right)$ for selecting the optimal among the neighboring nodes of the existing nodes $n_{i}$ is presented as

$$
\operatorname{FSF}\left(n_{i}, n_{j}\right)=\left\{\begin{array}{cc}
\frac{\left[P T\left(n_{i}, n_{j}\right)\right]^{\alpha}\left[E N\left(n_{j}\right)\right]^{\beta}\left[L P\left(n_{i}, n_{j}\right)\right]^{\gamma}\left[T R\left(n_{i}, n_{j}\right)\right]^{\delta}}{\sum n_{j} \in N B S\left(n_{i}\right)\left[P T\left(n_{i}, n_{j}\right)\right]^{\alpha}\left[\operatorname{EN}\left(n_{j}\right)\right]^{\beta}\left[\operatorname{LP}\left(n_{i}, n_{j}\right)\right]^{\gamma}\left[T R\left(n_{i}, n_{j}\right)\right]^{\delta}} & \text { if } n_{j} \in N B S\left(n_{i}\right) \\
0 & \text { otherwise }
\end{array}\right\} \ldots . .1
$$

Where, the set of neighboring nodes of $n_{i}$ is represented as NBS $\left(n_{i}\right)$, the concentration of pheromone deposited upon the path amid the nodes $n_{i} \& n_{j}$ is represented by PT $\left(n_{i}, n_{j}\right)$, the energy level of the neighbor node $n_{i}$ is represented by $\mathrm{EN}\left(n_{j}\right)$. The Trust rating of the neighbor node $n_{j}$ as provided by node $n_{i}$ is denoted by TR $\left(n_{i}, n_{i}\right)$. 
The link quality amid nodes $n_{i} \& n_{j}$, i.e., link probability is represented by $\operatorname{LP}\left(n_{i}, n_{j}\right)$. A measurement of the transmission link that is computed upon the basis of the past events occurring within the link is termed as the Expected Transmission Count, ETX.

Then the link probability $\operatorname{LP}\left(n_{i}, n_{j}\right)$ amid the nodes $n_{i} \& n_{j}$ is given by the expression:

$$
\operatorname{LP}\left(n_{i}, n_{j}\right)=\frac{1}{\operatorname{ETX}\left(n_{i}, n_{j}\right)} \ldots \ldots \ldots \ldots \ldots . . .2
$$

Where, $\alpha, \beta, \gamma, \delta$ are the parameters for controlling the prominence of pheromone course of the path, node energy level, link quality amid the nodes as well as rating of node trust. When $\alpha=\beta=\gamma=\delta=1$, the overall 4 parameters PT, EN, LP, TR are provided with similar significance for selecting the forwarder node. One could make $\alpha=\beta=\gamma=2, \delta=1$, similarly $\alpha=2, \beta=1, \gamma=\delta=2$ to raise importance of EN, Node Energy Level, $\alpha=2, \beta=\delta=2$, $\gamma=1$ to make importance of link quality more significant in the selection of forwarder node, when one is interesting to provide high significance.

Let, the initial energy of node $n_{j}$ is EI $\left(n_{j}\right)$ and the Remaining (Actual) Energy of node $n_{j}$ is ER $\left(n_{j}\right)$, then the description of Node Energy level, EN $\left(n_{j}\right)$ is as follows

$$
E N\left(n_{j}\right)=\frac{E R\left(n_{j}\right)}{E I\left(n_{j}\right.} \text { Where } E R\left(n_{j}\right)>E_{t h} \ldots \ldots 3
$$

\section{Pheromone Model}

It is recognised that number of pheromone evaluated for being placed upon the path throughout the return journey is not suitable for reflecting the path as optimum within the simulation period. Extended amounts of pheromone must be possessed by the stronger path while less number of pheromone or almost zero must be possessed by the weaker path. The selection of the stronger path is done always by the changes within the pheromone concentration amongst the competitive stronger paths on behalf of the selection.

In regard to this, the designing of the pheromone updated method is done by assuming the metrics and the collected forward ant throughout the journey from the source towards destination. After reaching the destination by the ant, the analyzing of the parameters is done by the forward ant.

\section{Result and Discussion}

NS-2.34 simulator is used to conduct the experiments and the experiment is conducted within 2 stages. Checking the sustainability of this plan is the primary stage and later the investigation is done deeply for assessing the delay as well as the throughput extensively.

40 mobile nodes are deployed within the network in the initial stage and the communication is initiated from the source towards the destination. The hop to hop communication takes place in this method and the distance is calculated upon the basis of the individual node's position. The measurement of the respective communication amid the users as well as the data flows is done. The transmission rate is known upon the basis of the pheromone values in this method. The maintenance of power as well as the delay on behalf of the respective nodes is done and the optimal path to selection of a routing is done in this method.

UDP connections are the connections amongst the mobile nodes and CBR (Constant Bit Rate) traffic within every communication channel is forwarded by this method. $512 \mathrm{~Kb} / \mathrm{s}$ is the CBR rate of the connections. $1500 \mathrm{~m} \mathrm{x}$ $1500 \mathrm{~m}$ is the scenario field size. A reviewed AODV routing protocol is used by this routing protocol where the integration of the ACO-DAEE, PDO-AODV approaches is performed.

Simulation table:

Table 2. Simulation table for network process

\begin{tabular}{|l|l|}
\hline PARAMETER & VALUE \\
\hline Application traffic & CBR \\
\hline Transmission rate & 5 packets/sec \\
\hline Radio range & $250 \mathrm{~m}$ \\
\hline
\end{tabular}




\begin{tabular}{|l|l|}
\hline Packet size & 1000 bytes \\
\hline Channel data rate & $2 \mathrm{Mbps}$ \\
\hline Maximum speed & $20 \mathrm{~m} / \mathrm{s}$ \\
\hline Simulation time & $10 \mathrm{secs}$ \\
\hline Number of nodes & 40 \\
\hline Area & $1500 \times 1500$ \\
\hline Routing protocol & AODV \\
\hline Routing methods & PDO-AODV, ACO-DAEE \\
\hline
\end{tabular}

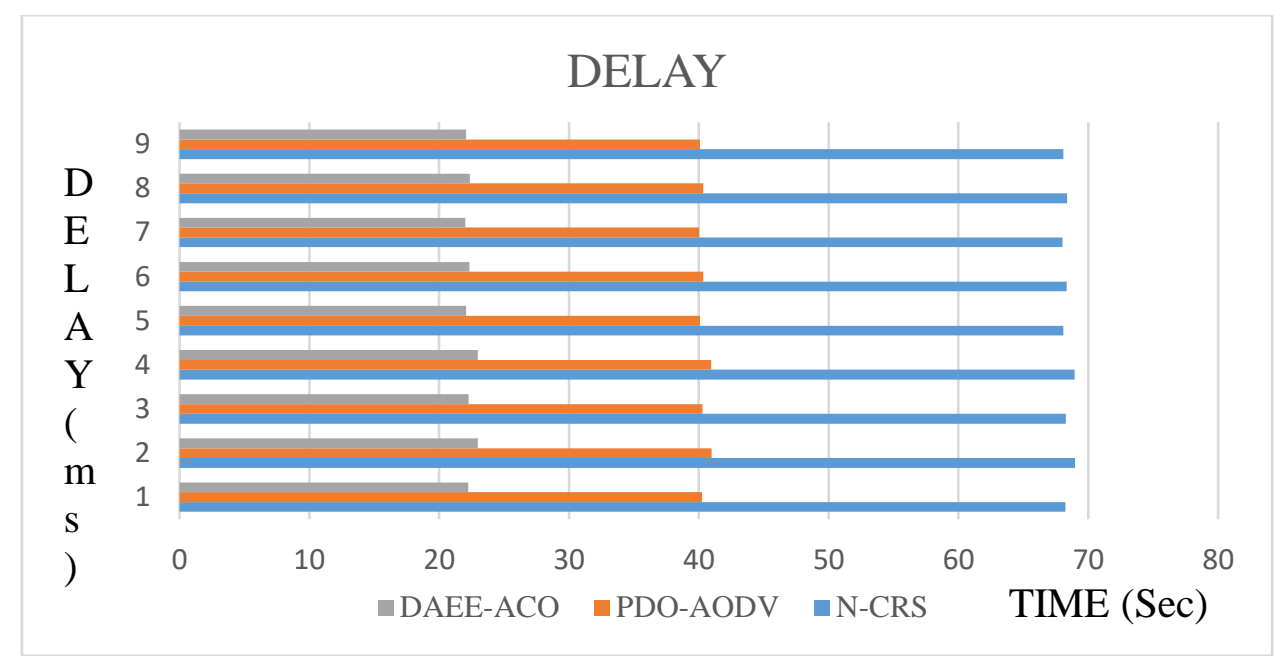

Figure 2. Delay

The End-to-End Delay of the network is shown in figure 2. The delay must be reduced within this method than the previous approaches for obtaining the optimum network performance. The delay within the network is reduced by proposing DAEE-ACO approach as it provides less delay compared to the earlier approaches such as PDOAODV, N-CRS.

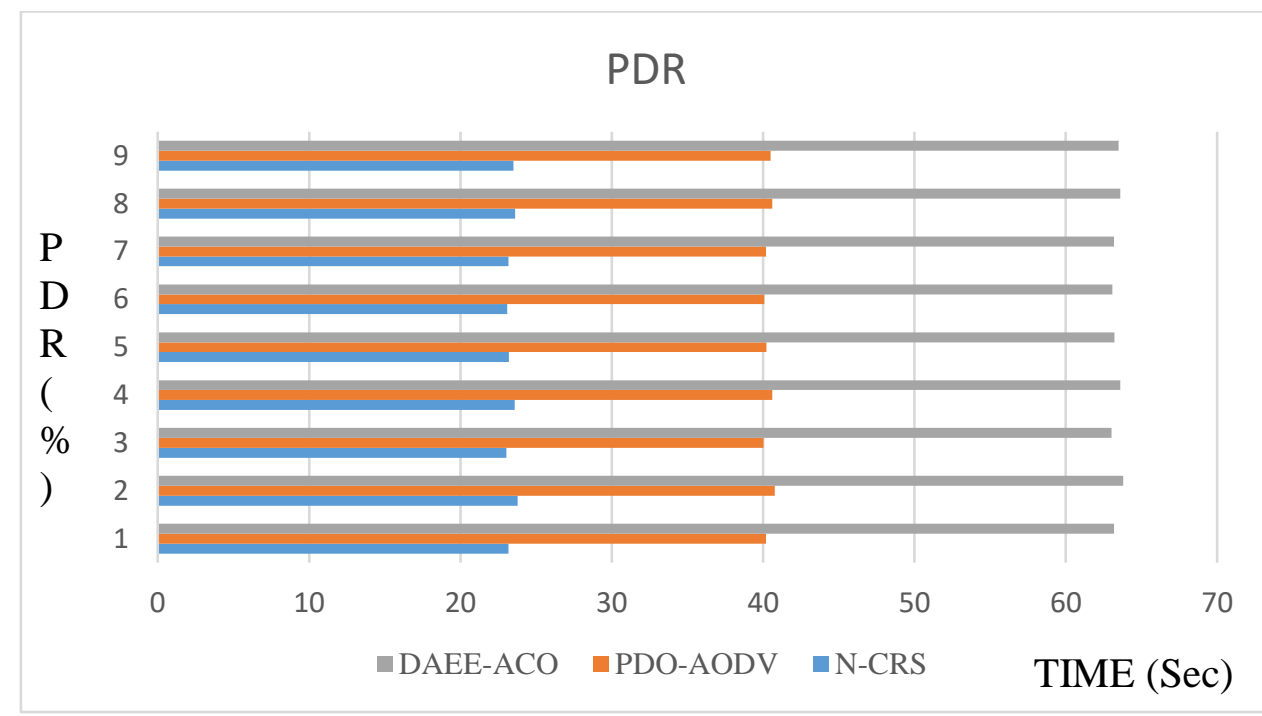

Figure 3. PDR

The PDR (Packet Delivery Ratio) of the network is shown in this figure. Higher Packet Delivery Ratio must be obtained in this method compared to the existing approaches for obtaining the improved network efficiency. The PDR within this network is increased by proposing DAEE-ACO approach and in turn it provides higher PDR compared to the previous techniques such as PDO-AODV, N-CRS. 


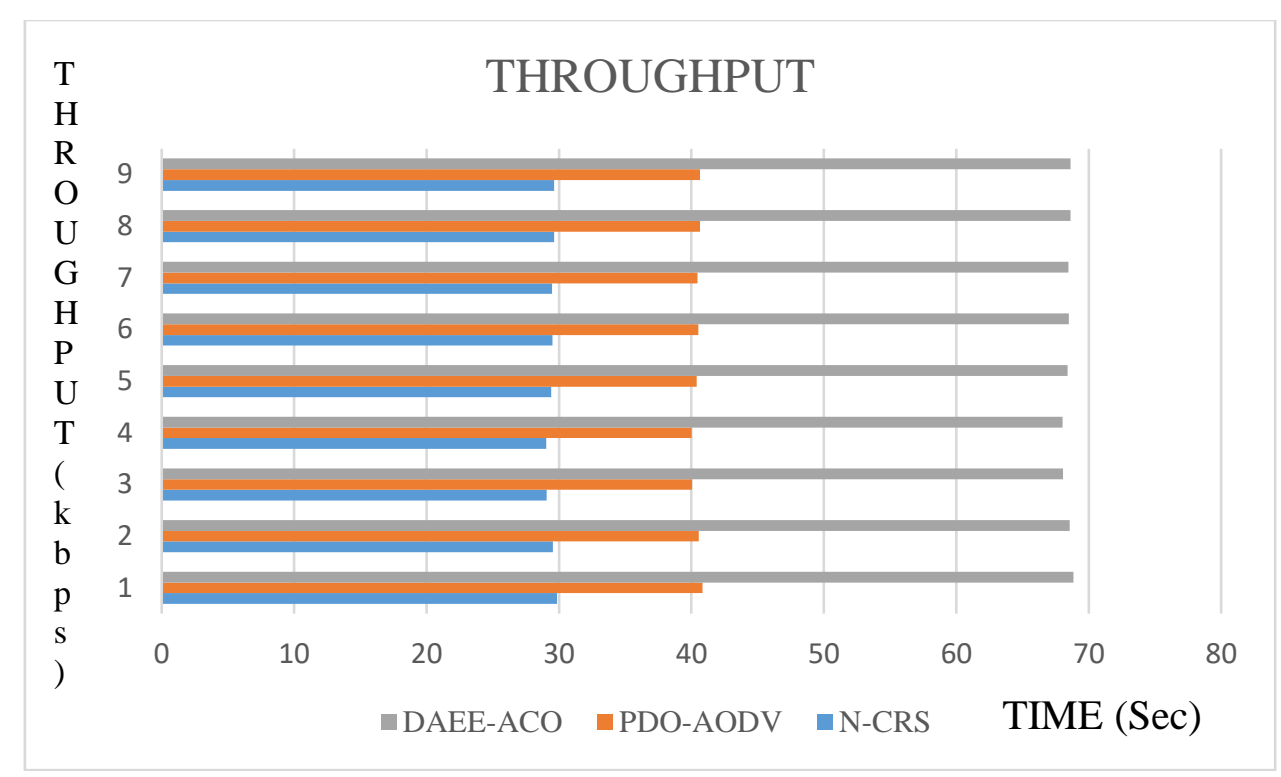

Figure 4. Throughput

The Throughput of the network is presented in the above figure. Higher throughput must be maintained in this method compared to the earlier techniques for obtaining the improved network performance. Compared to the existing techniques such as PDO-AODV \& N-CRS, the network throughput is increased by proposing DAEEACO approach and moreover it provides higher throughput delay.

\section{Conclusion}

The challenges of the effectiveness of power, selection of the nodes as well as the improper load balancing on behalf of the MANETs were surveyed by this method with the help of an interaction based cross layer mechanism. The optimization of the link cost upon the basis of the power and delay parameters for mitigating the huge problem that restores the resources of the earlier network are concentrated by the authors. Moreover, a friendship based handshaking functionality is utilized by them by means of a cross layer approach amid the data link layer as well as the network layer for accelerating the procedure of a routing layer. The data collection as well as the delay upon the routing is more in this method, therefore ACO (ant colony optimization) is proposed using the delay aware energy effective technique. The issues of the resource constraints of the ad-hoc network are solved by this method and it is shown in the simulation study that optimal performance is exhibited by it compared to the famous MANET protocols upon the basis of the equivalent cross layer technique. Simulation is performed by NS2 and it is demonstrated by the explorative results that ACO-DAEE approach is realistic on behalf of the discriminating delay aware nodes and the performance ratio within the network is improved.

\section{References}

1. M. Rath, B. Pati and B. K. Pattanayak, "Cross layer based QoS platform for multimedia transmission in MANET," 2017 11th International Conference on Intelligent Systems and Control (ISCO), Coimbatore, 2017, pp. 402-407, doi: 10.1109/ISCO.2017.7856026.

2. L. Lou and J. Fan, "A new anti-jamming reliable routing protocol for tactical MANETs," 2017 First International Conference on Electronics Instrumentation \& Information Systems (EIIS), Harbin, 2017, pp. 1-6, doi: 10.1109/EIIS.2017.8298766.

3. Elham Zamani and Mohammadreza Soltanaghaei, "The Improved Overhearing Backup AODV Protocol in MANET", Academic Editor: Rui Zhang, Received17 Aug 2015, Revised27 Dec 2015, Accepted03 Jan 2016, Published 24 Jan 2016, Volume 2016, Article ID 6463157, DoI: https://doi.org/10.1155/2016/6463157

4. Agbaria, A.; Gershinsky, G.; Naaman N. \& Shagin, K. Extrapolation-based and QoS-aware realtime communication in wireless mobile ad hoc networks. In the 8th IFIP Annual Mediterranean Adhoc Networking Workshop, Med-Hoc-Net 2009. pp.21-26. doi: 10.1109/MEDHOCNET.2009.5205201

5. Siva, K. \& P. Duraiswamy, K. A QoS routing protocol for mobile ad hoc networks based on the load distribution. In the IEEE International Conference on Computational Intelligence and Computing Research (ICCIC), 2010, pp.1-6. doi: 10.1109/ICCIC.2010.5705724 
6. Srivastava, S.; Daniel, A.K.; Singh, R. \& Saini, J.P. Energy efficient position based routing protocol for mobile ad hoc networks. In the IEEE International Conference on Radar Communication and Computing (ICRCC), 2012, pp.18-23. doi: 10.1109/ICRCC.2012.6450540

7. Ahmed, M.; Elmoniem, Abd; Ibrahim, Hosny M.; Mohamed, Marghny H. \& Hedar, Abdel Rahman. Ant colony and load balancing optimizations for AODV routing protocol. Int. J. Sensor Networks Data Commun., 2012, 1. doi: doi:10.4303/ijsndc/X110203

8. MadhanMohan, R. \& Selvakumar, K. Power controlled routing in wireless adhoc networks using cross layer approach. Egyptian Info. J., 2012, 13, 95-101. doi:10.1016/j.eij.2012.05.001

9. Ahmed, A.; Kumaran, T. Senthil S.; Syed, Abdul Syed \& Subburam, S. Cross-layer design approach for power control in mobile adhoc networks. Egyptian Info. J., 2015, 16(1), 1-7. doi:10.1016/j.eij.2014.11.001

10. Rath, M. \& Pattanayak, B.K. A methodical survey on real time applications in MANETS: Focussing on key issues. In International Conference on High Performance Computing and Applications (ICHPCA), 2014, pp.1-5. doi: 10.1109/ICHPCA.2014.7045301

11. Pattanayak, B.K. \& Rath, M. A mobile agent based intrusion detection system architecture for mobile adhoc networks. J. Comput. Sci., 2014, 10(6), 970-975. doi:10.3844/jcssp.2014.970.975

12. Han, G., Dong, Y., Guo, H., Shu, L., \& Wu, D. (2014). "Cross-layer optimized routing in wireless sensor networks with duty cycle and energy harvesting". Wireless Communications and Mobile Computing, 15(16), 1957-1981. doi:10.1002/wcm.2468.

13. M. Zhao, Y. Yang and C. Wang, "Mobile Data Gathering with Load Balanced Clustering and Dual Data Uploading in Wireless Sensor Networks," in IEEE Transactions on Mobile Computing, vol. 14, no. 4, pp. 770-785, 1 April 2015, doi: 10.1109/TMC.2014.2338315.

14. U. Sri Lakshmi, B. Srinivas Rao, "Secure Performance of intrusion detection system for manets using new digital signature" in IJIET, 7(1), on Jan 2016.

15. U. Sri Lakshmi, B. Srivas Rao, "Proficient Interference Exposure Expedients to Secure MANET from Occurrences" in IJAT, 8(4), on Oct 2017.

16. U. Sri Lakshmi, B. Srinivas Rao, "Mitigate the Routing overhead in WSN using new random walk detectors based approach" in Journal of Advanced Research Dynamic Control System International journal), issue 4, volume 10, on Oct 2018.

17. U. Sri Lakshmi, B. Srinivas Rao, “A Cross Layer Protocol to Improve Energy Efficiency and QoS in MANET" in Journal of Mechanics Continua and Mathematical Sciences, (ESCI Indexed journal, Web of Science), issue 1, volume 14, on Feb 2019.

18. U. Sri Lakshmi, B. Srinivas Rao, “An Overhead Aware Multipath Routing Protocol for Improving Relay Node Selection in MANET” in IJRTE (International journal), issue 1, volume 8, on May 2019.

19. Elhoseny, M., Shankar, K., \& Uthayakumar, J. Intelligent Diagnostic Prediction and Classification System for Chronic Kidney Disease, Nature Scientific Reports, July 2019. Press. DOI: https://doi.org/10.1038/s41598-019-46074-2.

20. Elhoseny, M., Bian, G. B., Lakshmanaprabu, S. K., Shankar, K., Singh, A. K., \& Wu, W. (2019). Effective features to classify ovarian cancer data in internet of medical things. Computer Networks, 159, 147-156.

21. Bayu Prabowo Sutjiatmo, Alfian Erwinsyah, E. Laxmi Lydia, K. Shankar, Phong Thanh Nguyen, Wahidah Hashim, Andino Maseleno, "Empowering Internet of Things (IoT) through BigData", international Journal of engineering and advanced technology(IJEAT), Vol.8, pg.938-942, August 2019.

22. Iswanto, I., Lydia, E. L., Shankar, K., Nguyen, P. T., Hashim, W., \& Maseleno, A. (2019). Identifying diseases and diagnosis using machine learning. International Journal of Engineering and Advanced Technology, 8(6 Special Issue 2), 978-981.

23. Lakshmanaprabu, S. K., Mohanty, S. N., Krishnamoorthy, S., Uthayakumar, J., \& Shankar, K. (2019). Online clinical decision support system using optimal deep neural networks. Applied Soft Computing, 81, 105487.

24. U. Srilakshmi, D. Yakobu, G. Jose Moses, P. Anusha, Dr. E. Laxmi Lydia, "Energy Efficient with load balancing Bee Ad-Hoc Routing Protocol for Manet" in Test Engineering \& Management (International journal), volume 82, on Jan 2020.

25. Uttej Kumar Nannapanenia, Dr. U. Srilakshmia, Alaparthi Sravyaa, Kavuru Tejaswia, “ClusterBased Collection point Energy Efficient Routing Protocol for the Mobile Sink in Wireless Sensor Network" in International Journal of Future Generation Communication and Networking, Vol. 13, No. 2, 2020 pp.787-796.

26. U. Srilakshmi, "Energy-Efficient Heterogeneous Optimization Routing Protocol for Wireless Sensor Network", in INSTRUMENTATION MESURE MÉTROLOGIE!, Vol. 19, No. 5, October, 2020, pp. 391-397. 